OPEN ACCESS

Edited by:

Hans-Georg Koch,

University of Freiburg, Germany

Reviewed by:

Ray A. Larsen,

Bowling Green State University,

United States

Kathryn Haley,

Grand Valley State University,

United States

*Correspondence:

Anchun Cheng

chenganchun@vip.163.com

Mafeng Liu

liumafengra@163.com

${ }^{\dagger}$ These authors have contributed equally to this work

Specialty section:

This article was submitted to Microbial Physiology and Metabolism,

a section of the journal

Frontiers in Microbiology

Received: 22 December 2020

Accepted: 13 July 2021

Published: 10 August 2021

Citation:

Zhang L, Huang L, Huang $M$, Wang $M$, Zhu $D$, Wang $M$, Jia $R$,

Chen S, Zhao X, Yang Q, Wu Y,

Zhang $S$, Huang J, Ou X, Mao $S$,

Gao Q, Tian B, Cheng A and Liu M

(2021) Effect of Nutritional

Determinants and TonB on

the Natural Transformation

of Riemerella anatipestifer.

Front. Microbiol. 12:644868.

doi: 10.3389/fmicb.2021.644868

\section{Effect of Nutritional Determinants and TonB on the Natural Transformation of Riemerella anatipestifer}

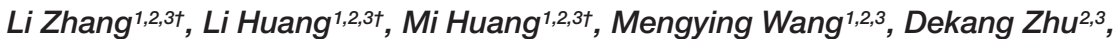 \\ Mingshu Wang ${ }^{1,2,3}$, Renyong Jia ${ }^{1,2,3}$, Shun Chen ${ }^{1,2,3}$, Xinxin Zhao ${ }^{1,2,3}$, Qiao Yang ${ }^{1,2,3}$, \\ Ying Wu ${ }^{1,2,3}$, Shaqiu Zhang ${ }^{1,2,3}$, Juan Huang ${ }^{1,2,3}$, Xumin Ou'1,2,3, Sai Mao ${ }^{1,2,3}$, Qun Gao ${ }^{1,2,3}$, \\ Bin Tian ${ }^{1,2,3}$, Anchun Cheng ${ }^{1,2,3 *}$ and Mafeng Liu ${ }^{1,2,3 *}$
}

\begin{abstract}
${ }^{1}$ Institute of Preventive Veterinary Medicine, Sichuan Agricultural University, Chengdu, China, ${ }^{2}$ Research Centre of Avian Disease, College of Veterinary Medicine of Sichuan Agricultural University, Chengdu, China, ${ }^{3}$ Key Laboratory of Animal

Disease and Human Health of Sichuan Province, Chengdu, China
\end{abstract}

Riemerella anatipestifer is a gram-negative bacterium that is the first naturally competent bacterium identified in the family Flavobacteriaceae. However, the determinants that influence the natural transformation and the underlying mechanism remain unknown. In this study, we evaluated the effects of various nutritional factors of the GCB medium [glucose, L-glutamine, vitamin $\mathrm{B} 1, \mathrm{Fe}\left(\mathrm{NO}_{3}\right)_{3}, \mathrm{NaCl}$, phosphate, and peptone], on the natural transformation of $R$. anatipestifer ATCC 11845. Among the assayed nutrients, peptone and phosphate affected the natural transformation of $R$. anatipestifer ATCC 11845 , and the transformation frequency was significantly decreased when phosphate or peptone was removed from the GCB medium. When the iron chelator $2,2^{\prime}$-dipyridyl (Dip) was added, the transformation frequency was decreased by approximately 100fold and restored gradually when $\mathrm{Fe}\left(\mathrm{NO}_{3}\right)_{3}$ was added, suggesting that the natural transformation of $R$. anatipestifer ATCC 11845 requires iron. Given the importance of TonB in nutrient transportation, we further identified whether TonB is involved in the natural transformation of $R$. anatipestifer ATCC 11845. Mutation of tonBA or tonBB, but not tbfA, was shown to inhibit the natural transformation of $R$. anatipestifer ATCC 11845 in the GCB medium. In parallel, it was shown that the tonBB mutant, but not the tonBA mutant, decreased iron acquisition in the GCB medium. This result suggested that the tonBB mutant affects the natural transformation frequency due to the deficiency of iron utilization.

Keywords: Riemerella anatipestifer, natural transformation, peptone, phosphate, iron, tonB

\section{INTRODUCTION}

Natural transformation is the process by which bacteria take up and integrate exogenous DNA into their genomes (Seitz and Blokesch, 2013). Most gram-negative bacteria use the type IV pilus family (T4P) to retract DNA into the periplasm, where one strand is degraded and the other is internalized into the cytoplasm through the ComEC transmembrane channel. 
Then, the internalized single-stranded DNA is bound by DprA. With the participation of RecA and ComM, the translocated strand replaces a chromosomal strand by recombination (Dubnau and Blokesch, 2019).

Transformation promotes the generation of new genetic traits and helps bacteria to adapt to new environmental conditions (Croucher et al., 2011). Thus, environmental determinants are important factors affecting natural transformation. Natural transformation has been observed and thoroughly studied in a wide variety of bacterial species, including Streptococcus pneumoniae (S. pneumoniae) (Lefrancois et al., 1998; Johnsborg and Havarstein, 2009; Straume et al., 2015), Neisseria (Facius and Meyer, 1993; Hamilton and Dillard, 2006; Zhang et al., 2013), Bacillus subtilis (B. subtilis) (Kosovich and Prozorov, 1990; Brautigam et al., 1997; Le et al., 2017), Haemophilus influenzae (H. influenzae) (Redfield, 1993; Mell et al., 2011), Vibrio cholerae (V. cholerae) (Frischer et al., 1990; Watve et al., 2014; Dalia et al., 2017), and Acinetobacter baylyi (A. baylyi) (Vaneechoutte et al., 2006; Merod and Wuertz, 2014; Utnes et al., 2015; Hulter et al., 2017; Leong et al., 2017; Suarez et al., 2017; Nero et al., 2018; Mantilla-Calderon et al., 2019). Although most bacteria possess competence genes, numerous conditions or signals trigger competence and are often species specific (Seitz and Blokesch, 2013). The expression of competence genes is influenced by the growth phase, cell density, metabolic activity, nutritional stress, and DNA damage (Johnsborg et al., 2007). For example, in S. pneumoniae and Helicobacter pylori, competence is induced by antibiotic stress or DNA damage (Prudhomme et al., 2006; Dorer et al., 2010).

Riemerella anatipestifer is a gram-negative bacterium that infects ducks, geese, turkeys, and other birds, and in ducklings, it can lead to a large number of deaths, resulting in huge economic losses (Wang et al., 2017). Currently, at least 21 different $R$. anatipestifer serotypes have been identified, among which, no cross-protection occurs (Helfer and Helmboldt, 1977; Leavitt and Ayroud, 1997), making eradication of this bacterium difficult. Previously, $R$. anatipestifer was described as a constitutive naturally transformable bacterium, although the genes that encode the natural transformation machinery were not identified completely (Liu et al., 2017b). Until now, several $R$. anatipestifer strains have been sequenced, and a comparison of the strains has revealed that the genomes are diverse (Wang et al., 2014; Liu J. et al., 2019). The genetic diversity could be caused by mutations, genomic rearrangements, and foreign DNA from the surrounding region. Additionally, $R$. anatipestifer exhibits resistance to multiple classes of antibiotics, including aminoglycosides, cephalosporins, chloramphenicol, lincosamides, macrolides, and nalidixic acid (Gyuris et al., 2017; Huang et al., 2017; Zhang et al., 2017; Luo et al., 2018), but the source of these resistance genes remains largely unknown. Thus, natural transformation can be hypothesized to play an important role in genomic diversity, the dissemination of antibiotic resistance, and evolution.

In this study, to understand the effect of nutritional determinants on the natural transformation of $R$. anatipestifer ATCC 11845, we investigated the efficiency of natural transformation under different nutrient conditions and found that peptone, phosphate, and iron influenced the natural transformation of $R$. anatipestifer ATCC 11845. Since the TonB of gram-negative bacteria is important for nutrient transportation, we further investigated the effect of TonB on the natural transformation of $R$. anatipestifer ATCC11845. R. anatipestifer encode ExbB-ExbD-TonB (TonB1 system), ExbB-ExbD-ExbD-TonB (TonB2 system), and TonB family protein (TbfA) for nutrient utilization (Liao et al., 2015; Liu et al., 2016). Here, TonB of TonB1 system and TonB2 system are re-termed as TonBA and TonBB, respectively. We found that the deletion of the ton $B A$ and ton $B B$ genes decreased the natural transformation frequency of R. anatipestifer ATCC 11845.

\section{MATERIALS AND METHODS}

\section{Bacterial Strains, Plasmids, and Primers}

The bacterial strains and plasmids used in this study are listed in Table 1. The primer sequences used in this study are listed in Table 2.

\section{Media and Growth Conditions}

The frozen bacterial stocks of $R$. anatipestifer ATCC 11845 were cultured on sheep blood agar plates for $16-18 \mathrm{~h}$ at $37^{\circ} \mathrm{C}$. Then, the cells from a single colony were inoculated to GC broth (GCB). GCB medium was prepared by supplementing $1 \mathrm{~L}$ of $\mathrm{H}_{2} \mathrm{O}$ with $1.5 \%$ peptone (Oxoid, China), $0.4 \% \mathrm{~K}_{2} \mathrm{HPO}_{4}$ (Sigma), $0.1 \% \mathrm{KH}_{2} \mathrm{PO}_{4}$ (Sigma), and $0.5 \% \mathrm{NaCl}$ (Sigma) plus $1 \%$ Kellogg's supplements I and 0.1\% Kellogg's supplements II as described in a previous study (Liu et al., 2017b). Kellogg's supplements I containing $40 \%$ glucose (Sigma), $1 \%$ L-glutamine (Sigma), and $0.002 \%$ vitamin B1; Kellogg's supplements II containing $20 \mathrm{mM} \mathrm{Fe}\left(\mathrm{NO}_{3}\right)_{3}$. Kellogg's supplements I was sterilized by a $0.45 \mu \mathrm{m}$ filter, others were sterilized using an autoclave. GCB agar plates were prepared by GCB supplementation with $1.5 \%$ agar. Escherichia coli (E. coli) strains were routinely cultured in LB liquid medium or on LB agar plates at $37^{\circ} \mathrm{C}$. Antibiotics were added at the following final concentrations: $1 \mu \mathrm{g} / \mathrm{ml}$ erythromycin (Erm), $1 \mu \mathrm{g} / \mathrm{ml}$ cefoxitin (Cfx), $20 \mu \mathrm{g} / \mathrm{ml}$ kanamycin (Kana), and $50 \mathrm{ng} / \mathrm{m} 1$ streptonigrin for R. anatipestifer and $100 \mu \mathrm{g} / \mathrm{ml}$ ampicillin (Amp) for E. coli.

\section{Preparation of Transforming DNA (tDNA)}

Transforming DNA (tDNA) for natural transformation experiments was amplified from the strain $R$. anatipestifer ATCC $11845 \triangle R A 0 C \_1551$ using the primers RAOC_1551 upP1 and RAOC_1551 downP2. The tDNA contains the upstream of RAOC_1551, the Erm antibiotic resistance cassette, and the downstream of RA0C_1551. The strain $R$. anatipestifer ATCC11845 $\triangle R A 0 C \_1551$ was constructed by transformation-mediated recombination in our previous study (Liu et al., 2017b). The fragments were purified using the TianGEN Extract II Kit (TIANGEN, Beijing, China). The concentration of 
TABLE 1 | Strains and plasmids used in this study.



$\mathrm{Erm}^{R}$ erythromycin resistance, $\mathrm{Amp}^{R}$ ampicillin resistance, $\mathrm{Km}^{R}$ kanamycin resistance, Cfx ${ }^{R}$ cefoxitin resistance. 
TABLE 2 | Primers used in this study.

\begin{tabular}{|c|c|c|}
\hline Primer & Organism & Sequence \\
\hline RAOC_1551 downP2 & RA ATCC 11845 & ПTCTTCGTIITTATCATAATATTTAAATAAGAAAAC \\
\hline tonBA upP2 & RA ATCC 11845 & CTAAAATCCTITATTGATTTGGCTAAGTTACTITCTTGTACGG \\
\hline tonBA downP1 & RA ATCC 11845 & CCGTACAAGAAAAGTAAACTTAGCCAAATCAATAAAAGGATTTTAG \\
\hline tonBA downP2 & RA ATCC 11845 & GGACTAGTCC GTTTAAGTCATTTAGCCTTCTAGC \\
\hline tonBB upP2 & RA ATCC 11845 & CTTGAACGAACTGGCTCTCCGCTCTGTCTTAGGTCATAAGCACCATAGGC \\
\hline ton $B B$ downP1 & RA ATCC 11845 & GCCTATGGTGCTTATGACCTAAGACAGAGCGGAGAGCCAGTTCGTTCAAG \\
\hline tonBB downP2 & RA ATCC 11845 & CCGCTCGAGCGGCAATAAAGACCACAGCATCTCCCGC \\
\hline tbfa upP1 & RA ATCC 11845 & CCGCTCGAGCGGCTATCTAGACATCAATGGTTCTATCGCTCAGCTAG \\
\hline tbfA upP2 & RA ATCC 11845 & GGTCAAAAATTGTAATTATTTATTGTCATAAAATTTAAAATTAATAC \\
\hline tbfa downP1 & RA ATCC 11845 & ATाTAAATITATGACAATAAATAATTACAATIITGACCAATC \\
\hline EXpheS P1 & pLMF03:pheS & ACGCGTCGACATTTCAAAAATTAACTTAAAACCACTG \\
\hline EXpheS P2 & pLMF03:pheS & GCTCTAGAGCCCTIIIITGTTACTTATAGCG \\
\hline 16s rRNA qRTP1 & RA ATCC 11845 & CGAAAGTGATAAGTTAGCCACCT \\
\hline 16s rRNA qRTP2 & RA ATCC 11845 & GCAGCACCTTGAAAATTGTCC \\
\hline dprA qRTP1 & RA ATCC 11845 & TCCGATGTTGAGGCAATTTG \\
\hline dprA qRTP2 & RA ATCC 11845 & TGCAAGTTTGGTTAGCGAGGTAG \\
\hline comEC qRTP1 & RA ATCC 11845 & CAATCCGAAATCTAACAGGCAAC \\
\hline comEC qRTP2 & RA ATCC 11845 & CGAAGTGGCTTGGCACATATT \\
\hline comM qRTP1 & RA ATCC 11845 & GTCGCCGCATCATACTATTTCC \\
\hline comM qRTP2 & RA ATCC 11845 & ATCCTCCAAAGGTTGCCTCATA \\
\hline tonBA qPCR $\mathrm{P} 1$ & RA ATCC 11845 & AAAGGAGGAAGTCGTAAGC \\
\hline RAOC_RS09840 qPCR P1 & RA ATCC 11845 & GAACTCACGAATATGCCAATACC \\
\hline RAOC_RS09840 qPCR P2 & RA ATCC 11845 & CCTATCCGTAACAGACCAACC \\
\hline
\end{tabular}

the fragments was measured by a NanoDrop 2000 spectrophotometer.

\section{Natural Transformation Assays}

The standard natural transformation assay was performed as described previously (Liu et al., 2017b). Briefly, the bacteria were grown to the exponential phase $\left(\mathrm{OD}_{600}=1.5-2.0\right)$ under aerobic conditions with shaking at $37^{\circ} \mathrm{C}$. Then, the bacteria were harvested and resuspended to an $\mathrm{OD}_{600}$ of 1 in the GCB medium. Then, $0.3 \mathrm{ml}$ of bacterial cells were transferred to sterilized tubes, and $1 \mu \mathrm{g}$ of tDNA fragments was added. The transformation was allowed to proceed at $37^{\circ} \mathrm{C}$ for $1 \mathrm{~h}$. Unabsorbed DNA was removed by washing, and then, the bacteria were plated on GCB plates containing $1 \mu \mathrm{g} / \mathrm{ml}$ Erm to obtain the transformants and on GCB plates to obtain the total viable bacteria, respectively. Transformation frequencies were calculated as the number of transformants divided by the total viable bacteria (Kristensen et al., 2012).

\section{The Effect of Different Components of GCB on Natural Transformation}

The exponential phase $R$. anatipestifer ATCC 11845 cultures $\left(\mathrm{OD}_{600}=1.5-2.0\right)$ were harvested, washed, and then resuspended to an $\mathrm{OD}_{600}$ of 1 in fresh GCB medium without one of the following: glucose, L-glutamine, vitamin $\mathrm{B} 1, \mathrm{Fe}\left(\mathrm{NO}_{3}\right)_{3}$, $\mathrm{NaCl}$, phosphate $\left(\mathrm{K}_{2} \mathrm{HPO}_{4}\right.$ and $\left.\mathrm{KH}_{2} \mathrm{PO}_{4}\right)$, or peptone. Following preincubation at $37^{\circ} \mathrm{C}$ for $1 \mathrm{~h}$ with shaking, the bacterial suspensions $(0.3 \mathrm{ml})$ were used to perform the natural transformation in these nutrient-limited medium. In parallel, the GCB medium without peptone or phosphate was supplemented with different concentrations of peptone $(0.5,1$ and $1.5 \%)$ or phosphate $(0.1-0.5 \%)$. Then, bacteria in exponential growth 
were harvested by centrifugation, washed, and resuspended to an $\mathrm{OD}_{600}$ of 1 in these media. After preincubation for $1 \mathrm{~h}$ at $37^{\circ} \mathrm{C}$ with shaking, $0.3 \mathrm{ml}$ of bacterial suspensions was used to perform the natural transformation in these media, respectively. In addition, sterile $\mathrm{H}_{2} \mathrm{O}$ was supplemented with $1.5 \%$ peptone, $0.5 \%$ phosphate $\left(0.4 \% \mathrm{~K}_{2} \mathrm{HPO}_{4}\right.$ and $\left.0.1 \% \mathrm{KH}_{2} \mathrm{PO}_{4}\right), 0.5 \% \mathrm{NaCl}$, $0.4 \%$ glucose, $0.01 \%$ L-glutamine, $0.00002 \% \mathrm{VB}_{1}, 20 \mu \mathrm{M} \mathrm{Fe}$ $\left(\mathrm{NO}_{3}\right)_{3}, 1.5 \%$ peptone plus $0.5 \%$ phosphate, or $0.5 \% \mathrm{NaCl}$ plus $0.5 \%$ phosphate, respectively. Then, the exponentially growing $R$. anatipestifer ATCC 11845 cells in GCB were collected by centrifugation, washed, and resuspended to an $\mathrm{OD}_{600}$ of 1 in these media. Following $1 \mathrm{~h}$ of preincubation, the bacterial suspensions $(0.3 \mathrm{ml})$ were used to perform natural transformation in these media, respectively.

\section{Natural Transformation Under Iron-Limited Conditions}

The bacteria were grown to an exponential phase in the GCB medium $\left(\mathrm{OD}_{600}=1.5-2.0\right)$ and then resuspended to an $\mathrm{OD}_{600}$

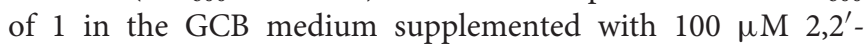
dipyridyl (Dip), which is able to chelate iron. In parallel, the bacterial suspensions (containing $100 \mu \mathrm{M}$ Dip) were divided into five fractions, and $0,2,4,20$, and $120 \mu \mathrm{M} \mathrm{Fe}\left(\mathrm{NO}_{3}\right)_{3}$ were added, respectively. Following $1 \mathrm{~h}$ of preincubation, $0.3 \mathrm{ml}$ of the bacterial suspension was used to perform natural transformation in these media, respectively.

\section{In vitro Growth Rate Determination}

The in vitro growth rates of $R$. anatipestifer ATCC 11845 under the experimental conditions described above were determined at $\mathrm{OD}_{600}$ in the spectrophotometer (Eppendorf Biophotometer, Germany). Briefly, the overnight cultured bacterial cells were inoculated into $20 \mathrm{ml}$ of the fresh medium at $\mathrm{OD}_{600}=0.05$ and incubated at $37^{\circ} \mathrm{C}$ with shaking at $180 \mathrm{rpm}$. The $\mathrm{OD}_{600}$ value was measured every $2 \mathrm{~h}$ for $14 \mathrm{~h}$. Cultures were diluted to bring the OD600 at $\sim 0.5$ when measured in $1.0 \mathrm{~cm}$ path length cuvettes.

\section{Construction of TonB Markerless Mutants and Complemented Strains in R. anatipestifer ATCC 11845}

The knockout plasmids pOES:tonBA, pOES:tonBB, and pOES:tbfA were constructed using the primers listed in Table 2. The construction of ton $B$ mutants was performed as described in a previous study (Liu et al., 2018). For complementation, the pLMF03 derivatives were transformed into relevant R. anatipestifer ATCC 11845 strains by conjugation as described previously (Liu et al., 2016).

\section{Sensitivity Assay Under Streptonigrin Stress}

The overnight cultured bacterial cells were inoculated into fresh $\mathrm{GCB}$ liquid medium at an $\mathrm{OD}_{600}$ of 0.05 . The cultures were incubated at $37^{\circ} \mathrm{C}$ with shaking $(180 \mathrm{rpm})$ until they reached an $\mathrm{OD}_{600}=1.5-2.0$, and then, the bacteria were harvested by centrifugation, washed, and resuspended in PBS to be adjusted to an $\mathrm{OD}_{600}=0.5$. The total viable bacteria of $1 \mathrm{ml}$ were counted by plating on the GCB plate. The final concentration of $50 \mathrm{ng} / \mathrm{ml}$ streptonigrin was added to $1 \mathrm{ml}$ of the bacterial suspension, and the mixture was incubated at $37^{\circ} \mathrm{C}$ for $30 \mathrm{~min}$. The incubated samples were washed and diluted serially to $10^{-5}$, $10^{-6}$, and $10^{-7}$ with PBS; $100 \mu \mathrm{l}$ of each dilution was spread onto GCB agar plates, and the viable bacteria were counted after incubation overnight at $37^{\circ} \mathrm{C}$. The survival rate was calculated by the number of surviving bacteria divided by the total number of viable bacteria.

\section{qRT-PCR}

$R$. anatipestifer ATCC 11845 was grown in the GCB medium starting at $\mathrm{OD}_{600}=0.05$ at $37^{\circ} \mathrm{C}$ with shaking at $180 \mathrm{rpm}$. When the cultures reached the exponential growth phase $\left(\mathrm{OD}_{600}=1.5-\right.$ 2.0), half of the bacteria were transferred to the GCB medium containing $100 \mu \mathrm{M}$ Dip. After $1 \mathrm{~h}$ of incubation with shaking, the bacterial cells in GCB and GCB with $100 \mu \mathrm{M}$ Dip were harvested for RNA extraction. Similarly, the exponentially bacterial cells were transferred to the GCB medium without phosphate, peptone, or phosphate plus peptone and incubated for $1 \mathrm{~h}$ at $37^{\circ} \mathrm{C}$. Then, the cells were collected for RNA extraction. For the analysis of the transcription level of ton $B s$, the wild-type, ton $B A$ mutant, ton $B B$ mutant, and tbf $A$ mutant strains were grown in the GCB medium until they reached the exponential growth phase $\left(\mathrm{OD}_{600}=1.5-2.0\right)$ and were harvested for total RNA extraction. Total RNA extraction was performed using the RNeasy Protect Bacteria Mini Kit (QIAGEN, Cat. Number 74524) as described in a previous study (Liu et al., 2016). cDNA was synthesized from each RNA sample according to our recent study (Liu et al., 2017b). Real-time PCR assays were conducted using the primers listed in Table 2. Quantitative PCR was performed on samples in triplicate using the standard curve protocol in which the calibration curve was generated using serial fivefold dilutions of $100 \mathrm{ng}$ of total cDNA. The RNA quantity was normalized using a probe specific for the 16S rRNA gene. Quantitative measurements were performed on biological samples in triplicate.

\section{Statistical Analysis}

Statistical analyses were performed using the GraphPad Prism 6 (GraphPad software, CA, United States) for Windows. Statistical significance was evaluated using the Student's $t$-test, one-way ANOVA, or two-way ANOVA. $p<0.05$ represents statistically significant differences. Error bars in all figures represent the standard deviations of three independent experiments.

\section{RESULTS}

\section{Natural Transformation of R. anatipestifer ATCC 11845 Under Nutrient-Restricted Conditions}

Since $R$. anatipestifer is naturally competent in all growth phases, and the transformation frequency is highest in the exponential growth phase (Liu et al., 2017b) and cell density do not play a significant role in competence development (data not shown), we suspected that the nutritional environment affects bacterial 
natural transformation. Next, we studied the effect of nutrient components in the GCB medium on the natural transformation of $R$. anatipestifer ATCC 11845, since the GCB medium promotes optimal competence and DNA uptake (Liu et al., 2017b, 2018; Liu M. et al., 2019).

First, we subjected cells from exponential phase cultures to resuspension in the GCB medium without glucose, L-glutamine, vitamin $\mathrm{B} 1, \mathrm{Fe}\left(\mathrm{NO}_{3}\right)_{3}, \mathrm{NaCl}$, phosphate, or peptone to perform natural transformation as described in section "Materials and Methods." As shown in Figure 1A, no significant differences in the transformation frequency of $R$. anatipestifer ATCC 11845 were observed in the GCB medium without glucose, L-glutamine, vitamin $\mathrm{B} 1, \mathrm{Fe}\left(\mathrm{NO}_{3}\right)_{3}$, or $\mathrm{NaCl}$. In contrast, the transformation frequency was significantly decreased when phosphate or peptone was removed (Figure 1A). To rule out the possibility of bacterial death, we also assessed the viability and growth in these nutrient-restricted media and found that $R$. anatipestifer ATCC 11845 survived well in these nutrient-restricted media during natural transformation assay (Figure 1B), but they did not grow in the GCB medium without vitamin B1, peptone, or phosphate (Figure 1B). Specifically, $R$. anatipestifer ATCC 11845 was observed to grow well in the GCB medium without $\mathrm{Fe}\left(\mathrm{NO}_{3}\right)_{3}$ (Figure 1C), suggesting that it was not an iron-limited medium. To create the iron-limited condition, the iron chelator 2,2'-dipyridyl (Dip) was added in the GCB medium without $\mathrm{Fe}\left(\mathrm{NO}_{3}\right)_{3}$. Thus, we compared the natural transformation frequency of $R$. anatipestifer ATCC 11845 in the GCB medium and GCB medium supplemented with $100 \mu \mathrm{M}$ Dip as described in section "Materials and Methods." As shown in Figure 1D, the transformation frequency decreased by approximately 100-fold when Dip was added. In addition to chelating $\mathrm{Fe}^{2+}$, Dip, as a divalent cation chelator, can also chelate other cations. To determine if the effect was caused by iron, we further performed natural transformation in GCB (100 $\mu \mathrm{M}$ Dip) supplemented with 0, 2, 4, 20, and $120 \mu \mathrm{M} \mathrm{Fe}\left(\mathrm{NO}_{3}\right)_{3}$, respectively. The result showed that the transformation frequency was gradually restored, suggesting that the natural transformation of $R$. anatipestifer ATCC 11845 requires iron. However, even though a high concentration of Fe $\left(\mathrm{NO}_{3}\right)_{3}$ was added, the transformation frequency could not be completely restored to the original level. It was suggested that Dip may chelate other cations related to natural transformation (Figure 1D). We also measured the effect of Dip on the viability and growth of $R$. anatipestifer ATCC 11845, and the results showed that Dip did not affect bacterial survival during natural transformation assay (Figure 1E). However, the bacterial cells did not grow in the GCB medium supplemented with $100 \mu \mathrm{M}$ Dip (data not shown). Thus, we chose to culture $R$. anatipestifer ATCC 11845 in the GCB medium containing $60 \mu \mathrm{M}$ Dip and found that the growth of bacteria was significantly inhibited but gradually restored when Fe $\left(\mathrm{NO}_{3}\right)_{3}$ was added (Figure 1F). These results indicated that phosphate, peptone, and iron affected the natural transformation of $R$. anatipestifer ATCC 11845 in the GCB medium. In contrast, depletion of vitamin B1, which is essential for the growth of $R$. anatipestifer ATCC 11845, did not have any effect on the natural transformation.
To further investigate the effect of phosphate and peptone on the natural transformation frequency of $R$. anatipestifer ATCC 11845, we transformed the tDNA into exponentially growing bacterial cells in the GCB medium depleted for or supplemented with different concentrations of phosphate or peptone, respectively. As shown in Figure 2A, the transformation ability of $R$. anatipestifer ATCC 11845 was gradually restored by the addition of 0.1 and $0.5 \%$ phosphate to the GCB medium without phosphate. To exclude the possibility that phosphate might affect the $\mathrm{pH}$, we measured the $\mathrm{pH}$ value of the phosphate-free GCB medium and GCB medium supplemented with different concentrations of phosphate and found that the $\mathrm{pH}$ did not change significantly in these media (data not shown). These results implied that the effect of phosphate on the natural transformation was not due to changes in the $\mathrm{pH}$. In addition, we also assessed the bacterial growth and the result showed that the growth of $R$. anatipestifer ATCC 11845 could be restored when phosphate was added, and when the concentration of phosphate was $0.5 \%$, the growth could return to its original level (Figure 2B). Similarly, as shown in Figure 2C, the lowest concentration of peptone tested $(0.5 \%)$ was able to restore the transformation ability of $R$. anatipestifer ATCC 11845. In addition, the growth was partially restored by adding 0.5 or $1 \%$ peptone and was completely restored by adding $1.5 \%$ peptone (Figure 2D).

We also measured whether iron, phosphate, and peptone have additive effects on the natural transformation of $R$. anatipestifer ATCC 11845. As shown in Supplementary Figure 1A, compared with the GCB medium without phosphate or peptone, the natural transformation frequency was decreased significantly when both phosphate and peptone were removed. Similarly, the natural transformation frequency was decreased significantly in the GCB medium without peptone when $100 \mu \mathrm{M}$ Dip was added (Supplementary Figure 1B).

\section{The Natural Competence of $R$. anatipestifer ATCC 11845 in Sterile $\mathrm{H}_{2} \mathrm{O}$ Supplemented With a Single Component of the GCB Medium}

Next, to further verify the function of GCB components in natural transformation, we investigated whether a single component of GCB medium has an effect on the natural transformation of $R$. anatipestifer ATCC 11845. As described in section "Materials and Methods," the transformation experiment was performed in sterile $\mathrm{H}_{2} \mathrm{O}$ and sterile $\mathrm{H}_{2} \mathrm{O}$ supplemented with the single component glucose, L-glutamine, vitamin B1, $\mathrm{Fe}\left(\mathrm{NO}_{3}\right)_{3}, \mathrm{NaCl}$, phosphate, or peptone, respectively. The results showed that the natural competence of $\mathrm{R}$. anatipestifer ATCC 11845 cannot occur in sterile $\mathrm{H}_{2} \mathrm{O}$ and sterile $\mathrm{H}_{2} \mathrm{O}$ supplemented with glucose, L-glutamine, vitamin $\mathrm{B} 1, \mathrm{Fe}\left(\mathrm{NO}_{3}\right)_{3}$, or phosphate, respectively. However, the transformants were detected after performing the natural transformation in sterile $\mathrm{H}_{2} \mathrm{O}$ supplemented with peptone or $\mathrm{NaCl}$ (Figure 3A), although only $20 \pm 5$ transformants per $\sim 10^{9}$ CFU were detected when the natural transformation was performed in sterile $\mathrm{H}_{2} \mathrm{O}$ supplemented with $\mathrm{NaCl}$. In parallel, the bacterial cells 

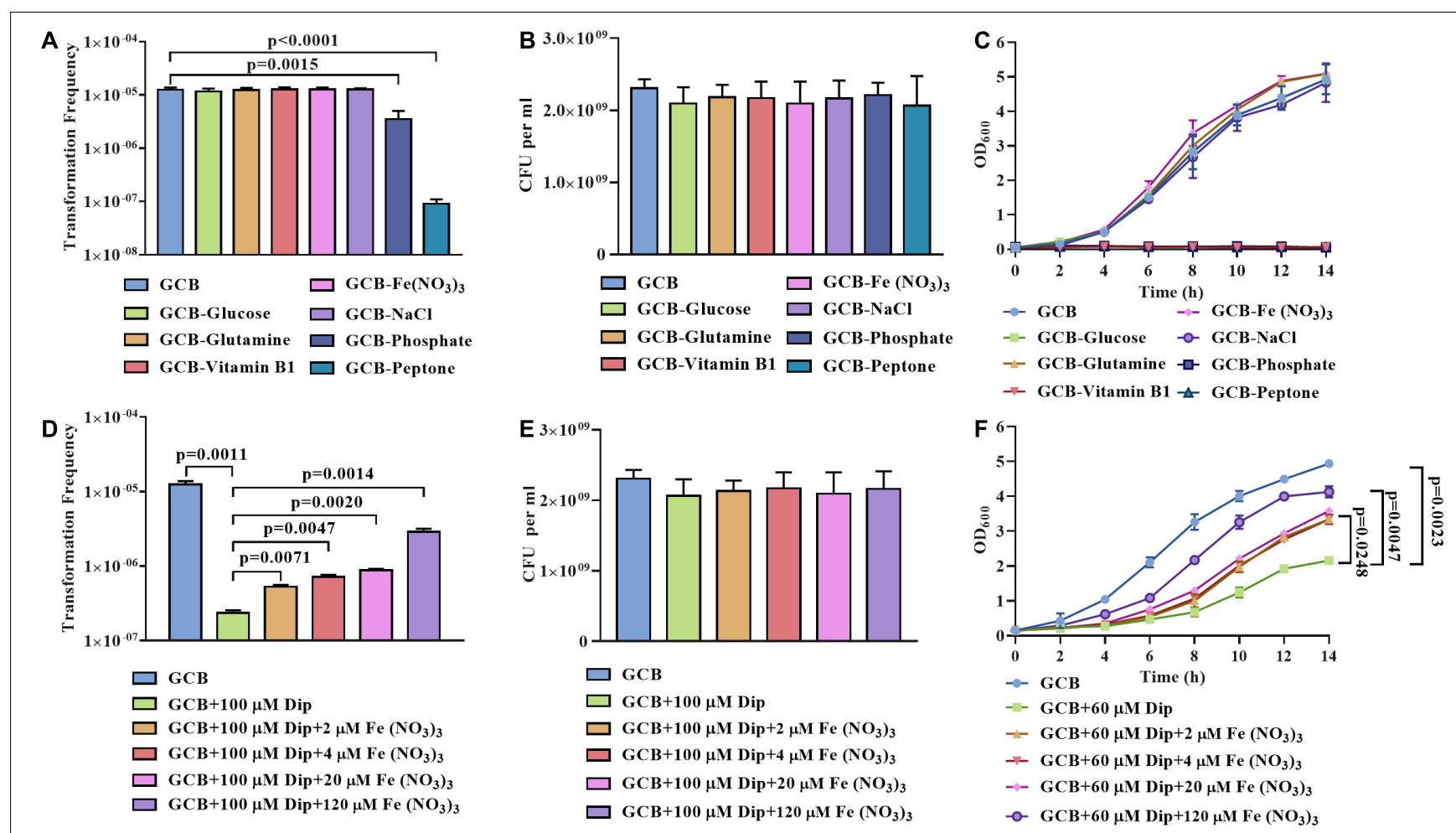

FIGURE 1 | Natural transformation assay of $R$. anatipestifer ATCC 11845 in nutrient-limited GCB medium. (A) The natural transformation frequency of R. anatipestifer ATCC 11845 in the GCB medium and GCB medium without glucose, L-glutamine, vitamin $\mathrm{B} 1, \mathrm{Fe}\left(\mathrm{NO}_{3}\right)_{3}, \mathrm{NaCl}, \mathrm{phosphate}$, and peptone, respectively. The bacteria were grown to the exponential phase in the GCB medium, then, they were resuspended to an $\mathrm{OD}_{600}$ of 1 in fresh $\mathrm{GCB}$ medium and $\mathrm{GCB}$ medium without one of the following: glucose, L-glutamine, vitamin $\mathrm{B} 1$, Fe $\left(\mathrm{NO}_{3}\right)_{3}, \mathrm{NaCl}$, phosphate $\left(\mathrm{K}_{2} \mathrm{HPO}_{4} \text { and } \mathrm{KH}_{2} \mathrm{PO}\right)_{4}$, and peptone, respectively. Following preincubation at $37^{\circ} \mathrm{C}$ for $1 \mathrm{~h}$ with shaking, the bacterial suspensions $(0.3 \mathrm{ml})$ were used to perform the natural transformation assay as described in section "Materials and Methods." (B) The input bacterial titer of $\sim 2.5 \times 10^{9} \mathrm{CFU}$ were suspended in 1 ml GCB medium and GCB without glucose, L-glutamine, vitamin B1, $\mathrm{Fe}\left(\mathrm{NO}_{3}\right)_{3}, \mathrm{NaCl}$, phosphate, and peptone, respectively. After $1 \mathrm{~h}$ of incubation at $37^{\circ} \mathrm{C}$, the viability of $R$. anatipestifer ATCC 11845 was determined. The number of survival bacteria did not change significantly $(p>0.05)$. (C) The growth curve of $R$. anatipestifer ATCC 11845 in the GCB medium and GCB medium without glucose, L-glutamine, vitamin $\mathrm{B} 1, \mathrm{Fe}\left(\mathrm{NO}_{3}\right)_{3}, \mathrm{NaCl}$, phosphate, and peptone, respectively. The bacteria were grown to the exponential phase in the GCB medium, then, they were resuspended in fresh GCB medium and GCB medium without one of the following: glucose, $\mathrm{L}$-glutamine, vitamin $\mathrm{B} 1$, Fe ( $\mathrm{NO})_{3}$, $\mathrm{NaCl}$, phosphate $\left(\mathrm{K}_{2} \mathrm{HPO}_{4}\right.$ and $\left.\mathrm{KH}_{2} \mathrm{PO}_{4}\right)$, and peptone, respectively, to an $\mathrm{OD}_{600}$ of 0.05 . The bacteria were cultured at $37^{\circ} \mathrm{C}$ with shaking, and the $\mathrm{OD}_{600}$ was measured every $2 \mathrm{~h}$ for $14 \mathrm{~h}$. (D) The effect of iron on the natural transformation frequency of $R$. anatipestifer ATCC 11845. The bacteria were grown to the exponential phase in the GCB medium, then, they were resuspended to an $\mathrm{OD}_{600}$ of 1 in fresh GCB medium, GCB medium containing $100 \mu \mathrm{M}$ Dip, and GCB medium containing $100 \mu \mathrm{M}$ Dip was supplemented with 2, 4, 20, and $120 \mu \mathrm{M} \mathrm{Fe}\left(\mathrm{NO}_{3}\right)_{3}$, respectively. Following preincubation at $37^{\circ} \mathrm{C}$ for $1 \mathrm{~h}$ with shaking, the bacterial suspensions $(0.3 \mathrm{ml})$ were used to perform the natural transformation assay as described in section "Materials and Methods." (E) The input bacterial titer of $\sim 2.5 \times 109$ CFU were suspended in $1 \mathrm{ml}$ of GCB medium, GCB medium containing $100 \mu \mathrm{M}$ Dip, GCB medium containing $100 \mu \mathrm{M}$ Dip supplemented with 2, 4, 20, and $120 \mu \mathrm{M}$ Fe(NO 3$)_{3}$, respectively. After $1 \mathrm{~h}$ of incubation at $37^{\circ} \mathrm{C}$, the viability of $R$. anatipestifer ATCC 11845 was determined. The number of survival bacteria did not change significantly ( $\left.p>0.05\right)$. (F) The growth curve of $R$. anatipestifer ATCC 11845 in the GCB medium, GCB medium containing $60 \mu M$ Dip, and GCB containing $60 \mu M$ Dip supplemented with $2,4,20$, and $120 \mu \mathrm{M} \mathrm{Fe}\left(\mathrm{NO}_{3}\right)_{3}$, respectively. The bacteria were grown to the exponential phase in the GCB medium, then, they were resuspended in fresh GCB medium, GCB medium containing $60 \mu \mathrm{M}$ Dip, and GCB containing $60 \mu \mathrm{M}$ Dip supplemented with 2, 4, 20, and 120 $\mu$ M Fe( $\left.\mathrm{NO}_{3}\right)_{3}$, respectively. The bacteria were cultured at $37^{\circ} \mathrm{C}$ with shaking, and the $\mathrm{OD}_{600}$ was measured every $2 \mathrm{~h}$ for $14 \mathrm{~h}$.

survived well in these media during natural transformation (Supplementary Figure 2).

Although phosphate plays a crucial role in the natural transformation of $R$. anatipestifer ATCC 11845 in the GCB medium, we did not observe the occurrence of natural transformation in sterile $\mathrm{H}_{2} \mathrm{O}$ supplemented with phosphate (Figure 3A). This prompted us to further investigate the natural transformation of $R$. anatipestifer ATCC 11845 in sterile $\mathrm{H}_{2} \mathrm{O}$ containing peptone or $\mathrm{NaCl}$ by adding phosphate. The results showed that the transformation frequency increased significantly after the addition of phosphate in sterile $\mathrm{H}_{2} \mathrm{O}$ with peptone or $\mathrm{NaCl}$ (Figure 3B). As a control, the bacterial cells survived well in these media during the natural transformation but did not have any growth (data not shown).

\section{Transcription of the Natural Competence Genes dprA, comEC, recA, and comM Under Nutrition-Restricted Conditions}

To further explore the mechanism affecting the natural transformation in $R$. anatipestifer ATCC 11845 in iron-limited GCB medium and in GCB medium without phosphate or peptone, we measured the transcription of conserved natural transformation genes in most competent bacteria, such as $\operatorname{dpr} A$, 


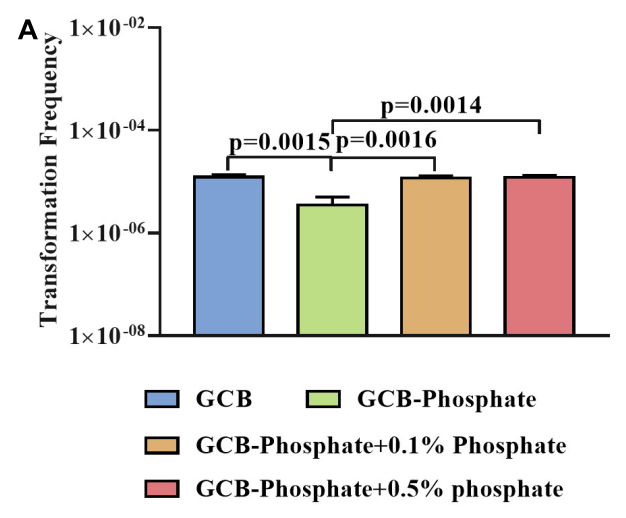

C

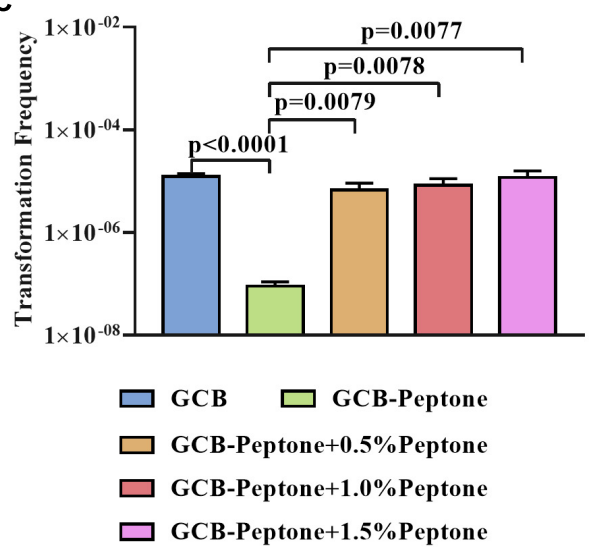

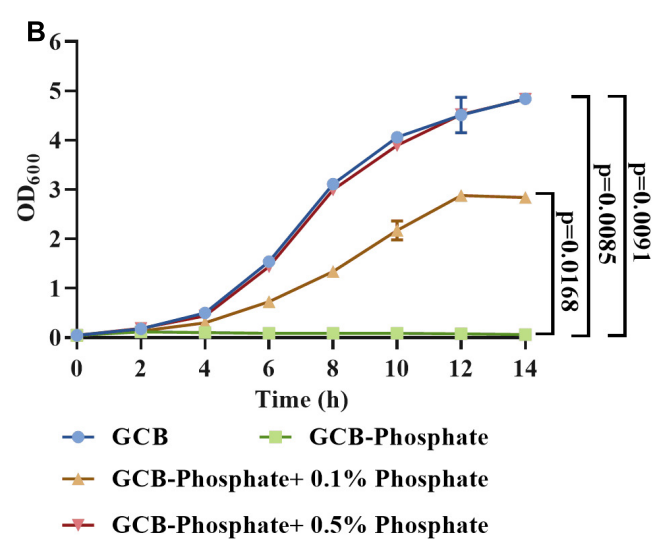

D

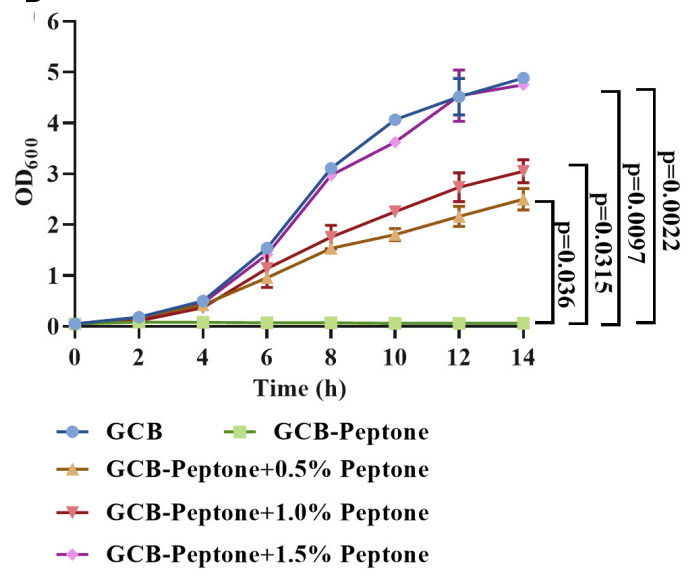

FIGURE 2 | The effect of phosphate and peptone on the natural transformation of $R$. anatipestifer ATCC 11845, respectively. (A) The natural transformation frequency of $R$. anatipestifer ATCC 11845 in the GCB medium, GCB medium without phosphate, and GCB medium without phosphate supplemented with 0.1 and $0.5 \%$ phosphate, respectively. The bacteria were grown to the exponential phase in the GCB medium, then, they were resuspended to an OD 600 of 1 in fresh GCB medium, GCB medium without phosphate, and GCB medium without phosphate supplemented with 0.1 and $0.5 \%$ phosphate, respectively. Following preincubation at $37^{\circ} \mathrm{C}$ for $1 \mathrm{~h}$ with shaking, the bacterial suspensions $(0.3 \mathrm{ml})$ were used to perform the natural transformation assay as described in section "Materials and Methods." (B) The growth curve of $R$. anatipestifer ATCC 11845 in GCB, GCB without phosphate, and GCB medium without phosphate supplemented with 0.1 and $0.5 \%$ phosphate, respectively. The bacteria were grown to the exponential phase in the GCB medium, then, they were resuspended in GCB, GCB without phosphate, and GCB medium without phosphate supplemented with 0.1 and $0.5 \%$ phosphate, respectively, to an OD600 of 0.05 . The bacteria were cultured at $37^{\circ} \mathrm{C}$ with shaking, and the $\mathrm{OD}_{600}$ was measured every $2 \mathrm{~h}$ for $14 \mathrm{~h}$. (C) The natural transformation frequency of $R$. anatipestifer ATCC 11845 in GCB, GCB without peptone, and GCB without peptone supplemented with $0.5,1$ and $1.5 \%$ peptone, respectively. The bacteria were grown to the exponential phase in the GCB medium, then, they were resuspended to an $\mathrm{OD}_{600}$ of 1 in fresh GCB medium, GCB medium without peptone, and GCB without peptone supplemented with $0.5,1$ and $1.5 \%$ peptone, respectively. Following preincubation at $37^{\circ} \mathrm{C}$ for $1 \mathrm{~h}$ with shaking, the bacterial suspensions (0.3 ml) were used to perform the natural transformation assay as described in section "Materials and Methods." (D) The growth curve of R. anatipestifer ATCC 11845 in GCB, GCB without peptone, and GCB without peptone supplemented with $0.5,1$, and $1.5 \%$ peptone, respectively. The bacteria were grown to the exponential phase in the GCB medium, then, they were resuspended in GCB, GCB without peptone, and GCB without peptone supplemented with $0.5,1$ and $1.5 \%$ peptone, respectively, to an OD 600 of 0.05 . The bacteria were cultured at $37^{\circ} \mathrm{C}$ with shaking, and the $\mathrm{OD}_{600}$ was measured every $2 \mathrm{~h}$ for $14 \mathrm{~h}$.

comEC, recA, and com $M$, which are involved in transporting ssDNA to the cytoplasm and facilitating RecA loading on internalized ssDNA and assisting homologous recombination (Johnston et al., 2014). The deletion of $d \operatorname{pr} A, \operatorname{comEC}$, recA, and com $M$ abolished the natural transformation of $R$. anatipestifer ATCC 11845 (Huang et al., 2019) (our unpublished data). As shown in Figure $\mathbf{4 A}$, the transcription of $d p r A$ and comEC was significantly downregulated (twofold) under iron-limited conditions, whereas that of recA and $\operatorname{com} M$ was not changed significantly under the same conditions, indicating that the decreased transformation frequency of $R$. anatipestifer ATCC 11845 under iron-limited conditions could be related to lower $d p r A$ and comEC expression. Interestingly, the levels of $d p r A$ and comEC transcripts in the GCB medium without phosphate were observed to be increased by two and threefold, respectively, and those of $\operatorname{rec} A$ and $\operatorname{com} M$ had almost no change (Figure 4B). This result suggested that the effect of phosphate on natural transformation was not caused by changes in $\operatorname{dpr} A, \operatorname{comEC}, \operatorname{rec} A$, or comM transcription levels. Moreover, in the GCB medium without peptone, only the transcription of $d \operatorname{pr} A$ was significantly 

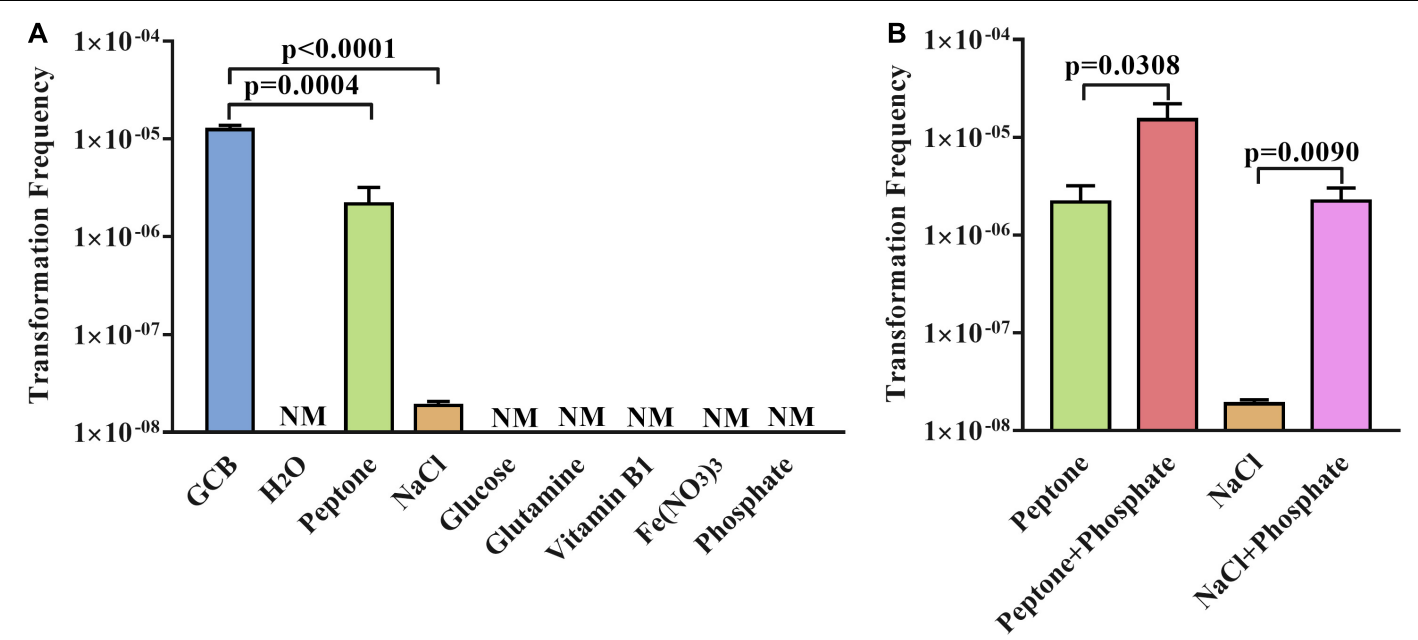

FIGURE 3 | The natural transformation frequency of $R$. anatipestifer ATCC 11845 in sterile $\mathrm{H}_{2} \mathrm{O}$ supplemented with single components or double components of GCB. (A) The transformation assay of $R$. anatipestifer ATCC 11845 in sterile $\mathrm{H}_{2} \mathrm{O}_{2}$ supplemented by a single component of the GCB medium. The bacteria were grown to the exponential phase in the GCB medium; then, they were resuspended to an $\mathrm{OD}_{600}$ of 1 in fresh GCB medium, sterile $\mathrm{H}_{2} \mathrm{O}$, sterile $\mathrm{H}_{2} \mathrm{O}$ supplemented with glucose, L-glutamine, vitamin $\mathrm{B} 1, \mathrm{Fe}\left(\mathrm{NO}_{3}\right)_{3}, \mathrm{NaCl}$, phosphate, and peptone, respectively. Following preincubation at $37^{\circ} \mathrm{C}$ for $1 \mathrm{~h}$ with shaking, the bacterial suspensions $(0.3 \mathrm{ml})$ were used to perform the natural transformation assay as described in section "Materials and Methods." (B) Natural transformation assay of R. anatipestifer ATCC 11845 in sterile $\mathrm{H}_{2} \mathrm{O}$ containing peptone, $\mathrm{NaCl}$, peptone and phosphate, $\mathrm{NaCl}$ and phosphate, respectively. The bacteria were grown to the exponential phase in the GCB medium, then, they were resuspended to an $\mathrm{OD}_{600}$ of 1 in sterile $\mathrm{H}_{2} \mathrm{O}$ supplemented with peptone, peptone and phosphate, $\mathrm{NaCl}$, and $\mathrm{NaCl}$ and phosphate, respectively. Following preincubation at $37^{\circ} \mathrm{C}$ for $1 \mathrm{~h}$ with shaking, the bacterial suspensions $(0.3 \mathrm{ml})$ were used to perform the natural transformation assay as described in section "Materials and Methods." NM represents no transformant.

downregulated (twofold), whereas that of $\operatorname{comEC}$, $\operatorname{rec} A$, and comM showed no statistically significant changes (Figure 4C). As evidence, the qRT-PCR results showed that the transcription of comEC was upregulated (twofold), whereas that of $\operatorname{dpr} A$, $\operatorname{rec} A$, and $\operatorname{com} M$ were not significantly different in the GCB medium without phosphate and peptone (Figure 4D). These findings suggested that iron, phosphate, and peptone may act through different mechanisms or at different stages of the natural transformation process.

\section{The Effect of TonB on the Natural Transformation of $\boldsymbol{R}$. anatipestifer ATCC 11845}

Since TonB of gram-negative bacteria is important for nutrient transportation, including iron (Noinaj et al., 2010), we further investigated whether TonB was involved in the natural transformation of $R$. anatipestifer ATCC 11845. In a previous study, it was shown that both TonBA and TonBB, but not TbfA, are required for hemin uptake in $R$. anatipestifer (Liao et al., 2015; Liu et al., 2016). As shown in Figure 5A, compared with the wild type, the natural transformation frequency was decreased significantly in the ton $B A$ and ton $B B$ mutants; however, the $t b f A$ mutation did not have any effect on the natural transformation of R. anatipestifer ATCC 11845. Particularly, it was observed that the ton $B B$ mutant inhibited the natural transformation more severely than the ton $B A$ mutant. Similarly, the ton $B A-\operatorname{ton} B B-t b f A$ triple mutant of $R$. anatipestifer ATCC 11845 exhibited a decreased natural transformation frequency than the single mutants, and the complementation of TonBA or TonBB, but not TbfA, was able to partially restore the natural transformation (Figure 5B). As a control, it was shown that $\operatorname{ton} B$ single mutant did not have any effect on the survival of the bacteria in the GCB medium during the natural transformation (Supplementary Figure 3).

Previous studies have established that the expression of the tonB genes of $R$. anatipestifer is not regulated by iron availability (Liu et al., 2017a). We then determined whether TonBB is more important or highly expressed than the other two TonBs. The transcription of ton $B A$, ton $B B$, and $t b f A$ in $R$. anatipestifer ATCC 11845 were measured and the results showed that ton $B B$ had the highest transcriptional level, and $t b f A$ had the lowest transcriptional level (Figure 6A). To identify whether the ton $B$ mutants influence the transcription of the other two tonBs, we further measured the transcription level of the three tonBs in each ton $B$ single mutant and found that the ton $B$ single mutants did not have any effect on the transcription of the other two tonBs (Figures 6B-D). It is suspected that TonBA and TonBB, but not TbfA, are involved in the natural transformation of R. anatipestifer ATCC 11845.

\section{The tonBB Mutant, but Not the tonBA Mutant, Damages the Iron Uptake of R. anatipestifer ATCC 11845}

TonBA and TonBB are involved in the natural transformation in the GCB medium, which can be reasoned by the deletion of $\operatorname{ton} B A$ or $\operatorname{ton} B B$ decreased the intracellular iron level in $R$. anatipestifer ATCC 11845. To verify this hypothesis, the sensitivity of the strains to streptonigrin, which exhibits an enhanced bacterial killing in the presence of iron (White and Yeowell, 1982), was compared. The results showed that R. anatipestifer ATCC11845 $\triangle$ tonBA did not exhibit a significant 

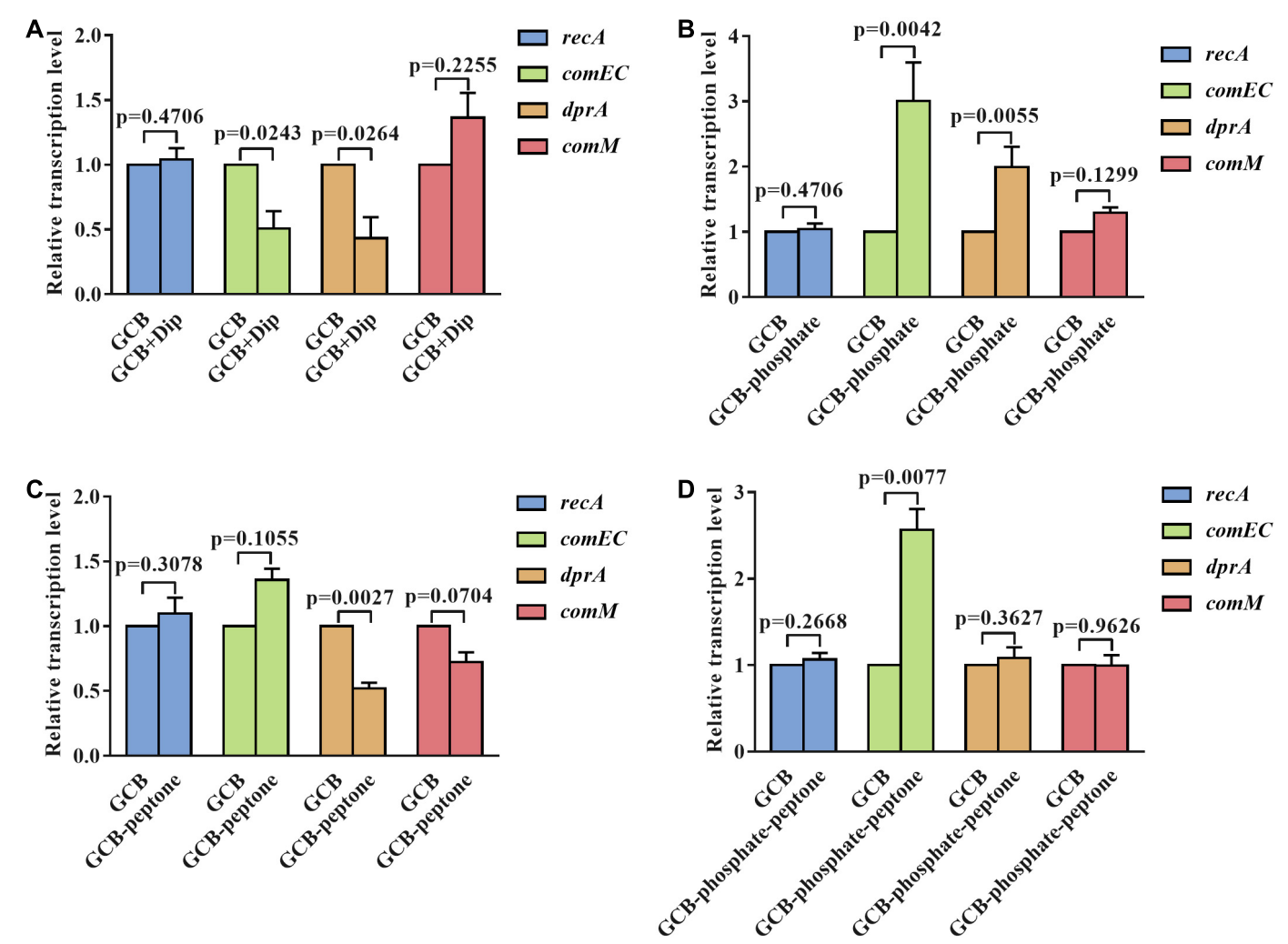

FIGURE 4 | The transcriptional levels of $d p r A$, comEC, recA, and comM in R. anatipestifer ATCC 11845 under different nutrient-limited conditions. (A) Quantitative real-time PCR analysis of the relative transcription of $d p r A, \operatorname{comEC}$, recA, and comM in $R$. anatipestifer ATCC 11845 in the GCB medium and GCB containing $100 \mu \mathrm{M}$ Dip as described in section "Materials and Methods." (B) Quantitative real-time PCR analysis of the relative transcription of dprA, comEC, recA, and comM in R. anatipestifer ATCC 11845 in GCB and GCB without phosphate as described in section "Materials and Methods." (C) Quantitative real-time PCR analysis of the relative transcription of $d p r A, \operatorname{comEC}$, recA, and comM in R. anatipestifer ATCC 11845 in GCB and GCB without peptone as described in section "Materials and Methods." (D) Quantitative real-time PCR analysis of the relative transcription of $d p r A$, comEC, recA, and comM in R. anatipestifer ATCC 11845 in GCB and GCB without phosphate and peptone as described in section "Materials and Methods." Fold changes were calculated by the ${ }_{\Delta} \Delta$ Ct method to consider the efficiency of the PCR for each target gene.
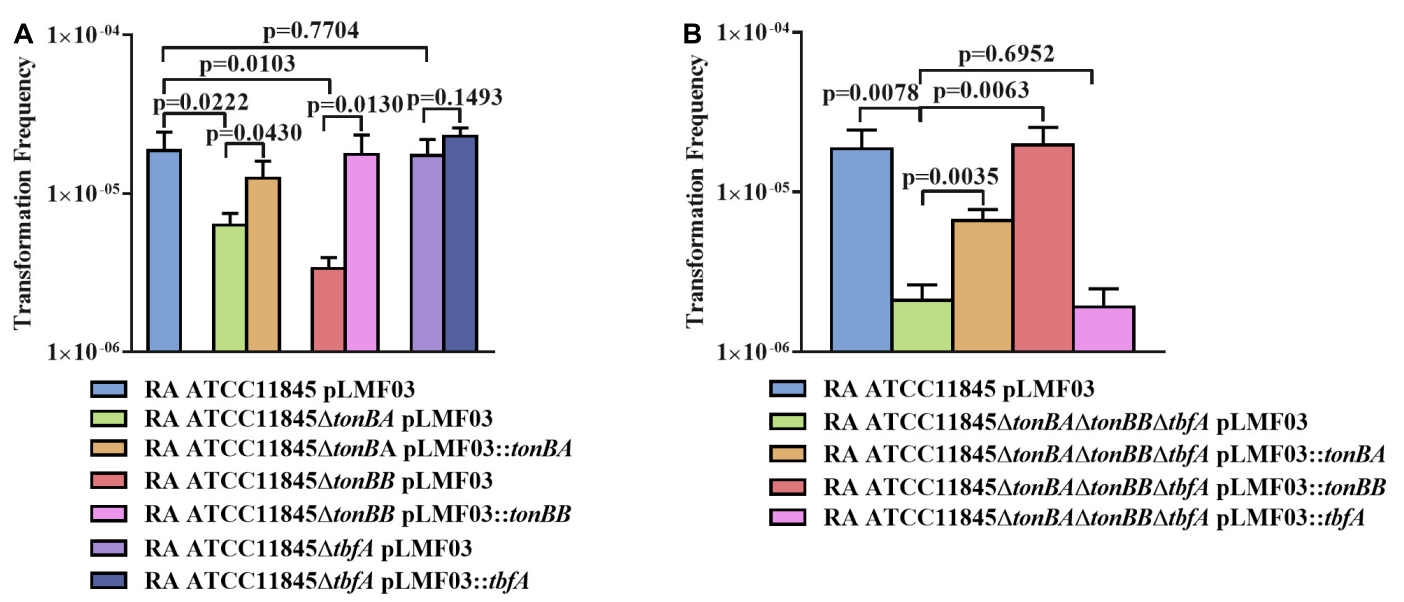

FIGURE 5 | The effect of tonB mutant on the natural transformation of $R$. anatipestifer ATCC 11845 in GCB. (A) The natural transformation frequency of the tonBA, tonBB, or tbfA mutants of $R$. anatipestifer ATCC 11845 in the GCB medium. (B) The natural transformation frequency of the tonBA-tonBB-tbfA triple mutant of R. anatipestifer ATCC 11845 and the single TonB complementation strain in the GCB medium. Natural transformation assay was performed using the standard method as described in section "Materials and Methods." 

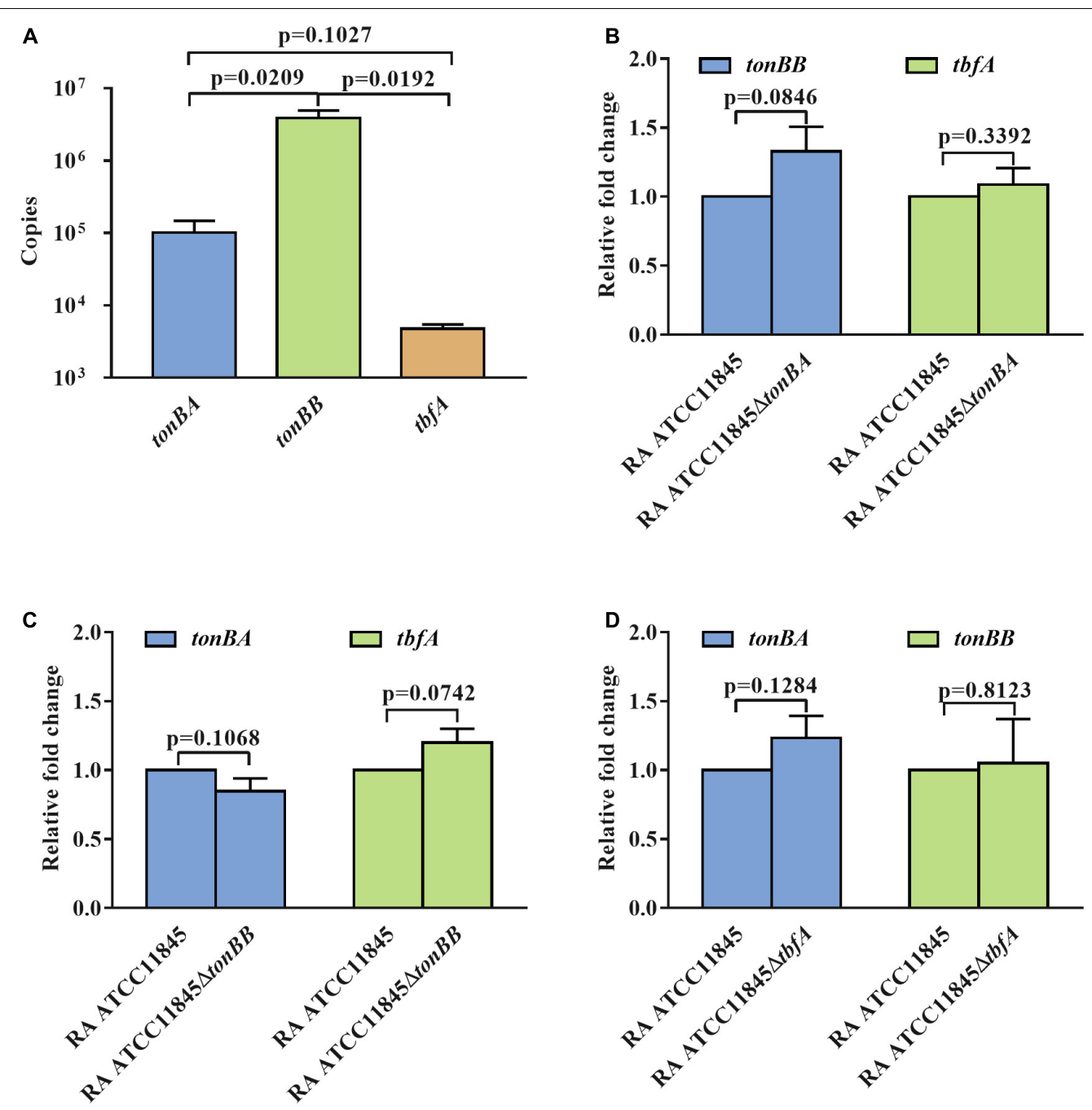

FIGURE 6 | The transcription level of the three tonBs in R. anatipestifer ATCC 11845 and its derivative strains. (A) The transcriptional copies number of the three tonBs in R. anatipestifer ATCC 11845. The standard curves of gene copies number and Ct values were established by amplifying tonBA, tonBB, and tbfA from plasmid pLMF03:tonBA, pLMF03:tonBB, and pLMF03:tbfA, respectively, using quantitative real-time PCR. Subsequently, the Ct values of three tonBs in $R$. anatipestifer ATCC 11845 were measured by amplifying tonBA, tonBB, and tbfA from cDNA of $R$. anatipestifer ATCC 11845. Finally, the transcriptional copies numbers were calculated according to the constructed standard curves. (B) The relative transcription level of the tonBB and tbfA in the tonBA mutant. (C) The relative transcription level of the ton $B A$ and $t b f A$ in the tonBB mutant. (D) The relative transcription level of the tonBA and tonBB in the tbfA mutant. The R. anatipestifer ATCC 11845, tonBA mutant, tonBB mutant, and tbfA mutant were grown in the GCB medium, respectively, until reaching the exponential growth phase and harvested for total RNA extraction and real-time quantitative PCR as described in section "Materials and Methods." Each experiment consisted of three biological replicate samples with three technical replicates each.

difference in survivability compared to the wild-type strain when treated with $50 \mathrm{~g} / \mathrm{ml}$ of streptonigrin (Figure 7). However, the survival rate of $R$. anatipestifer ATCC11845 $\triangle$ tonBB was significantly higher than that of the wild-type strain when treated with $50 \mathrm{ng} / \mathrm{ml}$ streptonigrin (Figure 7). The survival rate of the $\operatorname{ton} B A \operatorname{ton} B B$ double mutant did not change significantly compared to that of the $\operatorname{ton} B B$ single mutant when treated with $50 \mathrm{ng} / \mathrm{ml}$ streptonigrin (Figure 7). These results indicated that the $R$. anatipestifer ton $B B$ mutant, but not the ton $B A$ mutant, is defective in iron uptake.

If the concentration of intracellular iron is decreased, the putative iron uptake genes will be upregulated. Thus, we further measured the transcript levels of RAOC_RS09540 and RAOC_RS09840 in $R$. anatipestifer ATCC11845 $\triangle$ tonBA and $R$. anatipestifer ATCC11845 $\triangle$ tonBB, respectively, since their homologs were upregulated in $R$. anatipestifer $\mathrm{CH}-1$ under ironlimited conditions (Liu et al., 2017b; Huang M. et al., 2021). As shown in Supplementary Figure 4A, compared to that of the wild type, RAOC_RS09540 and RAOC_RS09840 had higher transcript levels in $R$. anatipestifer ATCC11845 $\triangle$ tonBB, but not in $R$. anatipestifer ATCC11845 $\triangle$ tonBA, when the bacteria were grown in the GCB medium. As a control, the transcripts of RAOC_RS09540 and RAOC_RS09840 in the wild type were higher in an iron-limited medium than in iron-rich medium 


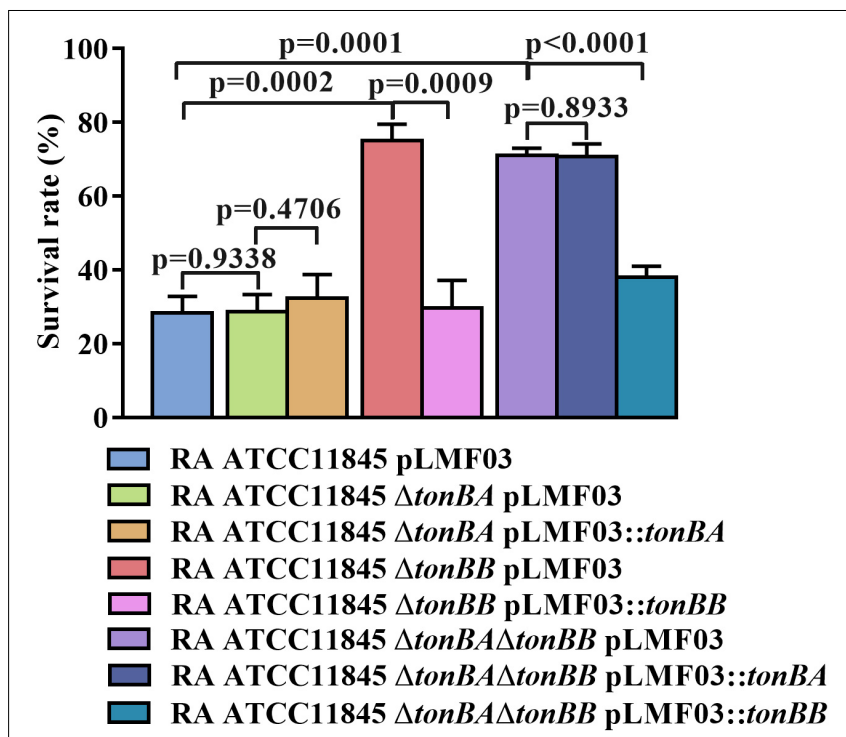

FIGURE 7 | The survival rate of $R$. anatipestifer ATCC11845 and its derivative strains when they were treated by streptonigrin. The bacteria were grown in the GCB medium until the exponential growth phase, then, harvested and resuspended in $\mathrm{PBS}$ to an $\mathrm{OD}_{600}=0.5$. The final concentration of $50 \mathrm{ng} / \mathrm{ml}$ Streptonigrin was added to $1 \mathrm{ml}$ of the bacterial suspension, and the mixture was incubated at $37^{\circ} \mathrm{C}$ for $30 \mathrm{~min}$. The survival rate was calculated as described in section "Materials and Methods."

(Supplementary Figure 4B). Taken together, these results suggest that the $\operatorname{ton} B B$ mutant, but not the ton $B A$ mutant, is defective in the iron uptake in $R$. anatipestifer ATCC11845 when grown in the GCB medium.

\section{DISCUSSION}

Natural transformation is a major mechanism of horizontal gene transfer in bacteria (Thomas and Nielsen, 2005). It has been shown that environmental cues play a crucial role in the occurrence of natural transformation (Seitz and Blokesch, 2013). As a newly identified natural competent bacterium, the factors that affect the natural transformation of $R$. anatipestifer are largely unknown. Here, we systematically examined the effects of nutrient factors on the natural transformation of R. anatipestifer ATCC 11845.

In a previous study, it was shown that the natural transformation of $R$. anatipestifer ATCC 11845 was constitutive and had a high transformation frequency in the exponential growth phase (Liu et al., 2017b). Thus, we hypothesize that the nutrition condition may affect the natural transformation of $R$. anatipestiferATCC11845. In this study, it was shown that the transformation frequencies were decreased significantly with the removal of phosphate or peptone and were gradually restored by the addition of phosphate or peptone, suggesting that phosphate and peptone affected the natural transformation of $R$. anatipestifer ATCC 11845. Interestingly, we found that the natural competence of $R$. anatipestifer ATCC 11845 could occur in sterile $\mathrm{H}_{2} \mathrm{O}$ supplemented with peptone or $\mathrm{NaCl}$, respectively, but not in phosphate. This result suggested that peptone and $\mathrm{NaCl}$ are able to induce the natural competence of $R$. anatipestifer ATCC 11845, while phosphate can enhance the natural transformation frequency but is not enough to initiate it. We further observed that the natural transformation of R. anatipestifer ATCC11845 also occurred in $\mathrm{H}_{2} \mathrm{O}$ supplemented by $\mathrm{NaHCO} 3$, but not $\mathrm{KCl}$ (data not shown), speculating that $\mathrm{Na}^{+}$has positive charges that neutralize the negativity of the phosphate backbone of the DNA to be transformed. Since peptone is a complex mixture and its nutritive source is largely dependent on the amino acid content, we speculated that peptone supplies amino acids for protein synthesis in cells that need to make the transformation machinery.

Iron is an essential element for the growth and survival of most bacteria since it functions in cellular processes such as respiration, oxidative stress resistance, and DNA synthesis (Andrews et al., 2003; Huang M. et al., 2021). In our study, the natural transformation was inhibited by adding $100 \mu \mathrm{M}$ Dip, and the transformation frequency was restored gradually by the supplementation with $\mathrm{Fe}\left(\mathrm{NO}_{3}\right)_{3}$. Dip is reported to be a chelator of divalent cations, which has been widely used in many studies to foster iron starvation (Lu et al., 2013; Yan et al., 2013; Karash and Kwon, 2018). It was suggested that the natural transformation of $R$. anatipestifer ATCC11845 could be affected by iron, implying that energy generation, as well as nucleotide synthesis, are important for the process of natural transformation. Notably, even though the high concentration of $\mathrm{Fe}\left(\mathrm{NO}_{3}\right)_{3}$ was added, the transformation ability cannot be completely restored to the original level. Our result was speculated that in addition to iron, the incorporation of Dip in the GCB medium may affect other divalent cation related to natural transformation. It was found that the member of Flavobacteriaceae, $R$. columbina was naturally competent and $\mathrm{Mg}^{2+}, \mathrm{Zn}^{2+}$, and $\mathrm{Mn}^{2+}$ promote the natural transformation frequency (Huang L. et al., 2021). Vitamin B1 is essential to nearly all cellular life, as it serves as a critical cofactor for many enzymes involved in carbohydrate metabolism (Sannino et al., 2018). In our study, we observed that R. anatipestifer ATCC 11845 could not grow in the GCB medium without vitamin $\mathrm{B} 1$, but the transformation frequency did not have any effect, suggesting that the natural transformability of $R$. anatipestifer may not be related to its growth.

Most gram-negative bacteria depend on the TonB complex to transport hemin and iron (Noinaj et al., 2010). Our previous studies showed that TonB proteins of $R$. anatipestifer are required for iron and hemin uptake (Liao et al., 2015; Liu et al., 2016, 2021). This prompted us to further determine whether TonB of $R$. anatipestifer ATCC 11845 was involved in the natural transformation. The results showed that the ton $B A$ or $\operatorname{ton} B B$ mutant decreased the natural transformation frequency of $R$. anatipestifer ATCC 11845 in the GCB medium. Moreover, compared with the ton $B A$ mutant, the ton $B B$ mutant decreased the natural transformation frequency is more significant. Streptonigrin is an antibiotic exhibiting irondependent bactericidal activity and has been used as an indirect approach to estimate free iron content in bacterial cells (Ganguly et al., 2018). In this study, we used streptonigrin sensitivity assay to evaluate the intracellular iron level of ton $B$ mutants and 
found that the $\operatorname{ton} B B$ mutant, but not the ton $B A$ mutant, was defective in the iron uptake in the GCB medium, speculating that the loss of ton $B B$ decreased the natural transformation frequency since it was damaged in iron utilization. Recently, it was reported that the TonB complex was required for the protein secretion in Myxococcus xanthus (Gomez-Santos et al., 2019). We speculated that the tonBA mutant may be damaged in the nutrient transportation related to natural transformation, such as peptone or phosphate, but this requires further study.

In summary, our study showed that peptone, phosphate, and iron influenced the transformation frequency of $R$. anatipestifer ATCC 11845 through a mutually independent pathway. In addition, our study also showed that TonBA and TonBB are involved in the natural transformation of $R$. anatipestifer. These observations provide further insights for understanding the natural transformation of R. anatipestifer.

\section{DATA AVAILABILITY STATEMENT}

The original contributions presented in the study are included in the article/Supplementary Material, further inquiries can be directed to the corresponding author/s.

\section{REFERENCES}

Andrews, S. C., Robinson, A. K., and Rodriguez-Quinones, F. (2003). Bacterial iron homeostasis. FEMS Microbiol. Rev. 27, 215-237. doi: 10.1016/S0168-6445(03) 00055-X

Brautigam, M., Hertel, C., and Hammes, W. P. (1997). Evidence for natural transformation of Bacillus subtilis in foodstuffs. FEMS Microbiol. Lett. 155, 93-98. doi: 10.1111/j.1574-6968.1997.tb12691.x

Croucher, N. J., Harris, S. R., Fraser, C., Quail, M. A., Burton, J., van der Linden, M., et al. (2011). Rapid pneumococcal evolution in response to clinical interventions. Science 331, 430-434. doi: 10.1126/science.1198545

Dalia, T. N., Hayes, C. A., Stolyar, S., Marx, C. J., McKinlay, J. B., and Dalia, A. B. (2017). Multiplex genome editing by natural transformation (MuGENT) for synthetic biology in Vibrio natriegens. ACS Synth. Biol. 6, 1650-1655. doi: 10.1021/acssynbio.7b00116

Dorer, M. S., Fero, J., and Salama, N. R. (2010). DNA damage triggers genetic exchange in Helicobacter pylori. PLoS Pathog. 6:e1001026. doi: 10.1371/journal. ppat. 1001026

Dubnau, D., and Blokesch, M. (2019). Mechanisms of DNA uptake by naturally competent bacteria. Annu. Rev. Genet. 53, 217-237. doi: 10.1146/annurevgenet-112618-043641

Facius, D., and Meyer, T. F. (1993). A novel determinant (comA) essential for natural transformation competence in Neisseria gonorrhoeae and the effect of a comA defect on pilin variation. Mol. Microbiol. 10, 699-712. doi: 10.1111/j. 1365-2958.1993.tb00942.x

Frischer, M. E., Thurmond, J. M., and Paul, J. H. (1990). Natural plasmid transformation in a high-frequency-of-transformation marine Vibrio strain. Appl. Environ. Microbiol. 56, 3439-3444. doi: 10.1128/AEM.56.11.3439-3444. 1990

Ganguly, T., Kajfasz, J., Miller, J., Rabinowitz, E., Galvão, L., Rosalen, P., et al. (2018). Disruption of a novel iron transport system reverses oxidative stress phenotypes of a dpr mutant strain of Streptococcus mutans. J. Bacteriol. 200:e00062-18. doi: 10.1128/jb.00062-18

Gomez-Santos, N., Glatter, T., Koebnik, R., Swiatek-Polatynska, M. A., and Sogaard-Andersen, L. (2019). A TonB-dependent transporter is required for

\section{AUTHOR CONTRIBUTIONS}

LZ, ML, and AC conceived and designed the research. LZ, LH, and $\mathrm{MH}$ performed the experiments and wrote the manuscript. $\mathrm{MeW}, \mathrm{DZ}, \mathrm{MiW}, \mathrm{RJ}, \mathrm{SC}$, and XZ participated in the experiments. QY, YW, SZ, and JH contributed the analysis tools. XO, SM, QG, and BT supervised the studies and corrected the manuscripts. All authors read and approved the final manuscript.

\section{FUNDING}

This work was supported by the National Natural Science Foundation of China (Grant No. 32072825, http://www.nsfc.gov. $\mathrm{cn} /$ ), the Sichuan Science and Technology Program (Grant No. 2020YJ0344), the China Agriculture Research System of MOF and MARA, and the Sichuan Veterinary Medicine and Drug Innovation Group of the China Agricultural Research System (Grant No. SCCXTD-2021-18).

\section{SUPPLEMENTARY MATERIAL}

The Supplementary Material for this article can be found online at: https://www.frontiersin.org/articles/10.3389/fmicb. 2021.644868/full\#supplementary-material

secretion of protease PopC across the bacterial outer membrane. Nat. Commun 10:1360. doi: 10.1038/s41467-019-09366-9

Gyuris, E., Wehmann, E., Czeibert, K., and Magyar, T. (2017). Antimicrobial susceptibility of Riemerella anatipestifer strains isolated from geese and ducks in Hungary. Acta Vet. Hung. 65, 153-165. doi: 10.1556/004.2017.016

Hamilton, H. L., and Dillard, J. P. (2006). Natural transformation of Neisseria gonorrhoeae: from DNA donation to homologous recombination. Mol. Microbiol. 59, 376-385. doi: 10.1111/j.1365-2958.2005.04964.x

Helfer, D. H., and Helmboldt, C. F. (1977). Pasteurella anatipestifer infection in turkeys. Avian Dis. 21, 712-715. doi: 10.2307/1589432

Huang, L., Liu, M., Zhu, D., Xie, L., Huang, M., Xiang, C., et al. (2021). Natural transformation of Riemerella columbina and its determinants. Front. Microbiol. 12:634895. doi: 10.3389/fmicb.2021.63 4895

Huang, L., Tian, X., Liu, M., Wang, M., Biville, F., Cheng, A., et al. (2019). DprA is essential for natural competence in Riemerella anatipestifer and has a conserved evolutionary mechanism. Front. Genet. 10:429. doi: 10.3389/fgene.2019.00429

Huang, L., Yuan, H., Liu, M. F., Zhao, X. X., Wang, M. S., Jia, R. Y., et al. (2017). Type B chloramphenicol acetyltransferases are responsible for chloramphenicol resistance in Riemerella anatipestifer, China. Front. Microbiol. 8:297. doi: 10. 3389/fmicb.2017.00297

Huang, M., Liu, M., Liu, J., Zhu, D., Tang, Q., Jia, R., et al. (2021). Functional characterization of Fur in iron metabolism, oxidative stress resistance and virulence of Riemerella anatipestifer. Vet. Res. 52:48. doi: 10.1186/s13567-02100919-9

Hulter, N., Sorum, V., Borch-Pedersen, K., Liljegren, M. M., Utnes, A. L., Primicerio, R., et al. (2017). Costs and benefits of natural transformation in Acinetobacter baylyi. BMC Microbiol. 17:34. doi: 10.1186/s12866-017-0953-2

Johnsborg, O., Eldholm, V., and Håvarstein, L. S. (2007). Natural genetic transformation: prevalence, mechanisms and function. Res. Microbiol. 158, 767-778. doi: 10.1016/j.resmic.2007.09.004

Johnsborg, O., and Havarstein, L. S. (2009). Regulation of natural genetic transformation and acquisition of transforming DNA in Streptococcus pneumoniae. FEMS Microbiol. Rev. 33, 627-642. doi: 10.1111/j.1574-6976.2009. 00167.x 
Johnston, C., Martin, B., Fichant, G., Polard, P., and Claverys, J. P. (2014). Bacterial transformation: distribution, shared mechanisms and divergent control. Nat. Rev. Microbiol. 12, 181-196. doi: 10.1038/nrmicro3199

Karash, S., and Kwon, Y. (2018). Iron-dependent essential genes in Salmonella Typhimurium. BMC Genomics 19:610. doi: 10.1186/s12864-018-4 986-1

Kosovich, P. B., and Prozorov, A. A. (1990). [Plasmid and chromosomal gene transfer in Bacillus subtilis and Escherichia coli in natural transformation]. Mol. Gen. Mikrobiol. Virusol. 29-31.

Kristensen, B. M., Sinha, S., Boyce, J. D., Bojesen, A. M., Mell, J. C., and Redfield, R. J. (2012). Natural transformation of Gallibacterium anatis. Appl. Environ. Microbiol. 78, 4914-4922. doi: 10.1128/AEM.00412-12

Le, S., Serrano, E., Kawamura, R., Carrasco, B., Yan, J., and Alonso, J. C. (2017). Bacillus subtilis RecA with DprA-SsbA antagonizes RecX function during natural transformation. Nucleic Acids Res. 45, 8873-8885. doi: 10.1093/nar/ gkx583

Leavitt, S., and Ayroud, M. (1997). Riemerella anatipestifer infection of domestic ducklings. Can. Vet. J. 38:113.

Lefrancois, J., Samrakandi, M. M., and Sicard, A. M. (1998). Electrotransformation and natural transformation of Streptococcus pneumoniae: requirement of DNA processing for recombination. Microbiology (Reading) 144(Pt 11), 3061-3068. doi: 10.1099/00221287-144-113061

Leong, C. G., Boyd, C. M., Roush, K. S., Tenente, R., Lang, K. M., and Lostroh, C. P. (2017). Succinate, iron chelation, and monovalent cations affect the transformation efficiency of Acinetobacter baylyi ATCC 33305 during growth in complex media. Can. J. Microbiol. 63, 851-856. doi: 10.1139/cjm-2017-0393

Liao, H., Cheng, X., Zhu, D., Wang, M., Jia, R., Chen, S., et al. (2015). TonB energy transduction systems of Riemerella anatipestifer are required for iron and hemin utilization. PLoS One 10:e0127506. doi: 10.1371/journal.pone.0127506

Liu, J., Zeng, Q., Wang, M., Cheng, A., Liu, M., Zhu, D., et al. (2019). Comparative genome-scale modelling of the pathogenic Flavobacteriaceae species Riemerella anatipestifer in China. Environ. Microbiol. 21, 2836-2851. doi: 10.1111/14622920.14635

Liu, M., Huang, M., Zhu, D., Wang, M., Jia, R., Chen, S., et al. (2017a). Riemerella anatipestifer identifying the genes responsible for iron-limited condition in CH-1 through RNA-Seq-based analysis. Biomed. Res. Int. 2017:8682057. doi: $10.1155 / 2017 / 8682057$

Liu, M., Huang, Y., Liu, J., Biville, F., Zhu, D., Wang, M., et al. (2018). Multiple genetic tools for editing the genome of Riemerella anatipestifer using a counterselectable marker. Appl. Microbiol. Biotechnol. 102, 7475-7488. doi: 10. 1007/s00253-018-9181-4

Liu, M., Liu, S., Huang, M., Wang, Y., Wang, M., Tian, X., et al. (2021). An exposed outer membrane haemin-binding protein facilitates haemin transport by a TonB-dependent receptor in Riemerella anatipestifer. Appl. Environ. Microbiol. 87:e0036721. doi: 10.1128/AEM.003 $67-21$

Liu, M., Tian, X., Wang, M., Zhu, D., Wang, M., Jia, R., et al. (2019). Development of a markerless gene deletion strategy using rpsL as a counterselectable marker and characterization of the function of RA0C_1534 in Riemerella anatipestifer ATCC11845 using this strategy. PLoS One 14:e0218241. doi: 10.1371/journal. pone. 0218241

Liu, M., Wang, M., Zhu, D., Wang, M., Jia, R., Chen, S., et al. (2016). Investigation of TbfA in Riemerella anatipestifer using plasmid-based methods for gene over-expression and knockdown. Sci. Rep. 6:37159. doi: 10.1038/srep37159

Liu, M., Zhang, L., Huang, L., Biville, F., Zhu, D., Wang, M., et al. (2017b). Use of natural transformation to establish an easy knockout method in Riemerella anatipestifer. Appl. Environ. Microbiol. 83:e00127-17. doi: 10.1128/AEM.001 27-17

Lu, F., Miao, S., Tu, J., Ni, X., Xing, L., Yu, H., et al. (2013). The role of TonB-dependent receptor TbdR1 in Riemerella anatipestifer in iron acquisition and virulence. Vet. Microbiol. 167, 713-718. doi: 10.1016/j.vetmic.2013.0 8.020

Luo, H. Y., Liu, M. F., Wang, M. S., Zhao, X. X., Jia, R. Y., Chen, S., et al. (2018). A novel resistance gene, $\ln u(\mathrm{H})$, conferring resistance to lincosamides in Riemerella anatipestifer CH-2. Int. J. Antimicrob. Agents 51, 136-139. doi: 10.1016/j.ijantimicag.2017.08.022
Mantilla-Calderon, D., Plewa, M. J., Michoud, G., Fodelianakis, S., Daffonchio, D., and Hong, P. Y. (2019). Water disinfection byproducts increase natural transformation rates of environmental DNA in Acinetobacter baylyi ADP1. Environ. Sci. Technol. 53, 6520-6528. doi: 10.1021/acs.est.9b0 0692

Mell, J. C., Shumilina, S., Hall, I. M., and Redfield, R. J. (2011). Transformation of natural genetic variation into Haemophilus influenzae genomes. PLoS Pathog. 7:e1002151. doi: 10.1371/journal.ppat.1002151

Merod, R. T., and Wuertz, S. (2014). Extracellular polymeric substance architecture influences natural genetic transformation of Acinetobacter baylyi in biofilms. Appl. Environ. Microbiol. 80, 7752-7757. doi: 10.1128/AEM.01984-14

Miller, V. L., and Mekalanos, J. J. (1988). A novel suicide vector and its use in construction of insertion mutations: osmoregulation of outer membrane proteins and virulence determinants in Vibrio cholerae requires toxR. J. Bacteriol. 170, 2575-2583. doi: 10.1128/jb.170.6.2575-2583. 1988

Nero, T. M., Dalia, T. N., Wang, J. C., Kysela, D. T., Bochman, M. L., and Dalia, A. B. (2018). ComM is a hexameric helicase that promotes branch migration during natural transformation in diverse Gram-negative species. Nucleic Acids Res. 46, 6099-6111. doi: 10.1093/nar/gky343

Noinaj, N., Guillier, M., Barnard, T. J., and Buchanan, S. K. (2010). TonBdependent transporters: regulation, structure, and function. Annu. Rev. Microbiol. 64, 43-60. doi: 10.1146/annurev.micro.112408.134247

Prudhomme, M., Attaiech, L., Sanchez, G., Martin, B., and Claverys, J. P. (2006). Antibiotic stress induces genetic transformability in the human pathogen Streptococcus pneumoniae. Science 313, 89-92. doi: 10.1126/science.1127912

Redfield, R. J. (1993). Evolution of natural transformation: testing the DNA repair hypothesis in Bacillus subtilis and Haemophilus influenzae. Genetics 133, 755-761. doi: 10.1093/genetics/133.4.755

Sannino, D., Kraft, C., Edwards, K., and Angert, E. (2018). Thiaminase I Provides a growth advantage by salvaging precursors from environmental thiamine and its analogs in Burkholderia thailandensis. Appl. Environ. Microbiol. 84:e01268-18. doi: 10.1128/aem.01268-18

Seitz, P., and Blokesch, M. (2013). Cues and regulatory pathways involved in natural competence and transformation in pathogenic and environmental Gram-negative bacteria. FEMS Microbiol. Rev. 37, 336-363. doi: 10.1111/j.15746976.2012.00353.x

Straume, D., Stamsas, G. A., and Havarstein, L. S. (2015). Natural transformation and genome evolution in Streptococcus pneumoniae. Infect. Genet. Evol. 33, 371-380. doi: 10.1016/j.meegid.2014.10.020

Suarez, G. A., Renda, B. A., Dasgupta, A., and Barrick, J. E. (2017). Reduced mutation rate and increased transformability of transposon-free Acinetobacter baylyi ADP1-ISx. Appl. Environ. Microbiol. 83:e01025-17. doi: 10.1128/AEM. 01025-17

Thomas, C. M., and Nielsen, K. M. (2005). Mechanisms of, and barriers to, horizontal gene transfer between bacteria. Nat. Rev. Microbiol. 3, 711-721. doi: $10.1038 /$ nrmicro1234

Utnes, A. L., Sorum, V., Hulter, N., Primicerio, R., Hegstad, J., Kloos, J., et al. (2015). Growth phase-specific evolutionary benefits of natural transformation in Acinetobacter baylyi. ISME J. 9, 2221-2231. doi: 10.1038/ismej.2015.35

Vaneechoutte, M., Young, D. M., Ornston, L. N., De Baere, T., Nemec, A., Van Der Reijden, T., et al. (2006). Naturally transformable Acinetobacter sp. strain ADP1 belongs to the newly described species Acinetobacter baylyi. Appl. Environ. Microbiol. 72, 932-936. doi: 10.1128/AEM.72.1.932-936.2006

Wang, M., Zhang, P., Zhu, D., Wang, M., Jia, R., Chen, S., et al. (2017). Identification of the ferric iron utilization gene B739_1208 and its role in the virulence of R. anatipestifer CH-1. Vet. Microbiol. 201, 162-169. doi: 10.1016/j. vetmic.2017.01.027

Wang, X., Liu, W., Zhu, D., Yang, L., Liu, M., Yin, S., et al. (2014). Comparative genomics of Riemerella anatipestifer reveals genetic diversity. BMC Genomics 15:479. doi: 10.1186/1471-2164-15-479

Wang, X., Zhu, D., Wang, M., Cheng, A., Jia, R., Zhou, Y., et al. (2012). Complete genome sequence of Riemerella anatipestifer reference strain. J. Bacteriol. 194, 3270-3271. doi: 10.1128/JB.00366-12

Watve, S. S., Bernardy, E. E., and Hammer, B. K. (2014). Vibrio cholerae: measuring natural transformation frequency. Curr. Protoc. Microbiol. 35, 6a.4.1-6a.4.12. doi: 10.1002/9780471729259.mc06a04s35 
White, J. R., and Yeowell, H. N. (1982). Iron enhances the bactericidal action of streptonigrin. Biochem. Biophys. Res. Commun. 106, 407-411. doi: 10.1016/0006-291x(82)91 125-1

Yan, Y., Waite-Cusic, J., Kuppusamy, P., and Yousef, A. (2013). Intracellular free iron and its potential role in ultrahigh-pressure-induced inactivation of Escherichia coli. Appl. Environ. Microbiol. 79, 722-724. doi: 10.1128/aem.022 02-12

Zhang, X., Wang, M. S., Liu, M. F., Zhu, D. K., Biville, F., Jia, R. Y., et al. (2017). Contribution of RaeB, a putative RND-type transporter to aminoglycoside and detergent resistance in Riemerella anatipestifer. Front. Microbiol. 8:2435. doi: 10.3389/fmicb.2017.0 2435

Zhang, Y., Heidrich, N., Ampattu, B. J., Gunderson, C. W., Seifert, H. S., Schoen, C., et al. (2013). Processing-independent CRISPR RNAs limit natural transformation in Neisseria meningitidis. Mol. Cell 50, 488-503. doi: 10.1016/j.molcel.2013.05.001
Conflict of Interest: The authors declare that the research was conducted in the absence of any commercial or financial relationships that could be construed as a potential conflict of interest.

Publisher's Note: All claims expressed in this article are solely those of the authors and do not necessarily represent those of their affiliated organizations, or those of the publisher, the editors and the reviewers. Any product that may be evaluated in this article, or claim that may be made by its manufacturer, is not guaranteed or endorsed by the publisher.

Copyright (c) 2021 Zhang, Huang, Huang, Wang, Zhu, Wang, Jia, Chen, Zhao, Yang, Wu, Zhang, Huang, Ou, Mao, Gao, Tian, Cheng and Liu. This is an open-access article distributed under the terms of the Creative Commons Attribution License (CC BY). The use, distribution or reproduction in other forums is permitted, provided the original author(s) and the copyright owner(s) are credited and that the original publication in this journal is cited, in accordance with accepted academic practice. No use, distribution or reproduction is permitted which does not comply with these terms. 